\title{
北京城市蝴蝶蜜源植物网络特征及重要蜜源植物识别
}

\author{
韩 丹 $^{1,2}$, 王 成 ${ }^{1,2, *}$, 殷鲁秦 ${ }^{1,2}$
}

1 中国林业科学研究院林业研究所,国家林业和草原局林木培育重点实验室,北京 100091

2 国家林业和草原局城市森林研究中心, 北京 100091

摘要:物种间相互作用网络研究能为物种多样性的保护、城市生态系统稳定性的维持提供指导。基于群落水平的城市蝴蝶蜜源 植物互作网络的研究较少, 对城市蝴蝶蜜源网络结构缺乏深人认识。研究在国内城市生态系统中构建蝴蝶蜜源网络, 并探讨不 同类型植物对网络特征的影响。2020 年 6-9 月,在北京 26 个城市公园中记录访花蝴蝶和蜜源植物物种及互作频次,采用交互 多样性 $(I D)$ 、交互均匀性 $(I E)$ 、专业化程度 $\left(H 2^{\prime}\right)$ 定量化生态网络结构特征,采用 Kruskal-Wallis 秩和检验和变差分解分析不同 生长型、起源、栽培方式的植物类型对网络结构的影响差异。采用物种的伙伴多样性 $(P D)$ 和专业化程度 $\left(d^{\prime}\right)$ 识别重要蜜源植 物。研究结果表明: (1) 北京城市公园中 22 种蝴蝶与 81 种开花植物的交互作用,形成趋于泛化的生态网络结构; (2) 不同生长 型及不同起源的植物-蝴蝶网络的交互多样性及专业化程度有显著差异, 草本及乡土植物对丰富网络中交互多样性和支持专业 性更高的蝴蝶物种具重要作用,而植物的栽培方式对蜜源网络结构影响较小; (3) 伙伴多样性高且专业化程度高的植物可被视 为重要蜜源植物。基于蝴蝶多样性保护的目标, 在城市生态系统中, 绿色空间应注重构建乡土草本植物群落,优先选择重要蜜 源植物。我们的发现印证了蝴蝶-蜜源植物生态网络方法作为联结生态研究和城市绿地实践管理的有效工具, 能为城市生态系 统中生物多样性保护提供科学策略,具有重要意义。

关键词:生态二元网络;蝴蝶多样性;蜜源植物;植物类型;相互作用; 城市生态系统

\section{Characteristics of butterfly-nectar plant network in Beijing's urban parks and identifying important nectariferous plant species}

\author{
HAN Dan ${ }^{1,2}$, WANG Cheng ${ }^{1,2, *}$, YIN Luqin ${ }^{1,2}$ \\ 1 Research Institute of Forestry, Chinese Academy of Forestry, Key Laboratory of Tree Breeding and Cultivation, National Forestry and Grassland \\ Administration, Beijing 100091, China \\ 2 Urban Forest Research Center, National Forestry and Grassland Administration, Beijing 100091, China
}

\begin{abstract}
Study on ecological networks about species interactions provides a basis for species diversity protection, and it is beneficial for the maintenance of urban ecosystem stability. However, urban ecological network study between butterfly and plant based on community level still leaves a huge blank, therefore, the knowledge of butterfly-nectar plant network structure needs an in-depth understanding. To our knowledge, ours is the first study focusing on butterfly-nectar plant network in urban ecosystems in China and evaluating the effects of different type of plants on butterfly-nectar plant network. In this study, we surveyed flower visiting butterfly species and nectariferous plant species and the interaction frequency in 26 urban parks in Beijing, China from June to September in 2020. The butterfly-nectar plant bipartite network was built. We identified five quantitative metrics on two hierarchical levels (network and species) to describe network patterns and species' interaction. Metrics on network level were interaction diversity ( $I D)$, interaction evenness ( $I E)$ and network
\end{abstract}

基金项目:中央级公益性科研院所基本科研业务费专项资金（CAFYBB2020ZB008）；国家自然科学基金青年科学基金项目(31800608)

收稿日期:2020-12-18; 接收日期:2021-09-28

* 通讯作者 Corresponding author.E-mail: wch8361@163.com 
specialization $\left(H 2^{\prime}\right)$. Moreover, we analyzed the effect of different vegetation types on the network structure differences, including different type of growth form, origin, and cultivation plant, using Kruskal-Wallis test and variance partitioning analysis. Metrics on species level were partner diversity $(P D)$ and species specialization $\left(d^{\prime}\right)$ for identifying important nectariferous plant species that provide a key and central role to the stability of the structure of network. The results are as follows: (1) 22 species of butterfly and 81 species of flowering plants of interaction were observed and the butterfly-nectar plant network tended to be generalized. (2) The interaction diversity and specialization of two growth form plant-butterfly network were significantly different $(P<0.01)$, and same to of two origin plant. Furthermore, herbs and native plants played an important role in maintaining the interaction diversity and specialized butterfly species in the network. Interestingly, cultivation type of plants had little effect on nectar network structure. (3) Plants with high partner diversity and high degree of specialization could be regarded as important nectariferous plant species. Consequently, in order to protect butterfly diversity, native herbaceous plant community should be constructed in green space of urban ecosystem, and important nectariferous plant species should be given top priority to grow. Our findings confirm that the butterfly-nectar plant ecological network can provide a scientific strategy for biodiversity conservation in urban ecosystems. It could be an effective tool to link ecology research with urban green space practice management.

Key Words: ecological bipartite network; butterfly diversity; nectariferous plant; vegetation type; interaction; urban ecosystem

物种间相互作用是地球生态系统中生物多样性的重要组成部分之一 ${ }^{[1]}$ 。城市生物多样性的保护不仅需 要关注物种本身, 更有必要研究物种间相互作用的关系。作为生态系统的有效描述方式之一, 生态网络包括 节点和节点之间的链接 ${ }^{[2]}$, 节点可以是个体、物种、功能群甚至群落等,链接是指节点之间的联系。生态二元 网络方法 (bipartite network) 不仅只关注节点和单一的生物多样性指标,更强调物种间相互作用的模式 ${ }^{[3]}$ 。生 态网络的分析有助于确定群落的组成和稳健性、资源分配以及组成物种之间的联系 ${ }^{[2,4]}$ 。我们需要在应用生 态学中更明确地纳人生态网络方法, 以改善当前基于物种或以栖息地为中心的生态保护方法, 更好地管理和 保护面临环境变化的生物多样性 ${ }^{[5]}$ 。

植物与传粉动物之间存在直接或间接的联系 ${ }^{[2]}$, 蝴蝶是自然群落中常见的传粉动物。蝴蝶与蜜源植物 间的相互作用可形成蝴蝶-蜜源植物生态网络。蝴蝶作为环境指示物种之一,保护城市蝴蝶多样性是维持城 市生态系统健康的重要行动之一。从群落水平的角度, 采用生态二元网络中网络层级的指标可以评估城市蝴 蝶蜜源植物网络的稳定性和专业化程度 ${ }^{[6]}$ 。蝴蝶多样性常随植物多样性的增加而增加 ${ }^{[7]}$, 在城市环境中, 局 部生境资源仍是维持蝴蝶种群的决定性因素之一 ${ }^{[8-9]}$ 。而城市公园的园林管理活动往往大规模地消除部分 富含花蜜的自然更新植物, 又引进较多的外来植物 ${ }^{[10]}$, 花卉种植更替频繁, 从而可能影响蝴蝶与蜜源植物群 落结构。在被传粉动物的访问过程中,乡土植物与外来植物也存在竞争 ${ }^{[6]}$, 而不同更新方式和生长型植物 ${ }^{[11]}$ 对维持传粉者种群方面也各有特定价值。有必要进一步了解不同类型植物对蜜源网络结构的影响,这对维持 城市生态系统中稳健的传粉网络结构有重要意义 ${ }^{[12]}$ 。

与此同时,物种可能会从与其互惠伙伴的互动中受益,部分物种也会依赖于互惠伙伴生存。蝴蝶的功能 作用发挥与其对开花植物传粉、在寄主植物上产卵和发育有关 ${ }^{[11]}$, 同时开花植物的花蜜作为蝴蝶的食物, 有 利于其种群的维持和增长。有必要进一步评价蝴蝶和蜜源植物互相作用的特点,评估其抵抗环境变化风险的 能力, 因此需采用物种层级的指标识别特定物种的专业化程度 ${ }^{[13]}$ 。特定蝴蝶物种对特定植物物种的依赖性 不同,往往形成相互作用强度不对称的蝴蝶蜜源植物网络。通过城市蝴蝶和蜜源植物的生态二元网络研究, 明确蝴蝶的植物偏好并识别重要蜜源植物物种, 能为蝴蝶多样性的维持提供依据, 对制定城市生态系统中的 物种保护策略有重要意义 ${ }^{[14]}$ 。

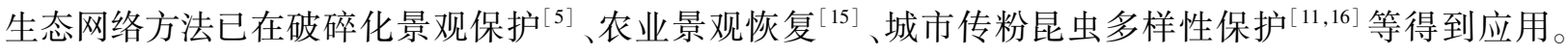


但我国在群落水平上的传粉网络结构研究较少, 且主要集中在自然生态系统 ${ }^{[1,17-18]}$, 同时在城市生态系统中 的蝴蝶植物互作网络的研究几乎空白。因此,本研究构建北京城市蝴蝶蜜源网络,用生态二元网络的结构属 性作为指标来评估保护目标, 识别重要蜜源植物物种,并分析不同生长型、起源以及栽培方式的植物对蝴蝶蜜 源网络结构的影响差异, 可为城市地区营建蝴蝶栖息地提供植物选择的科学指导, 有利于城市蝴蝶多样性保 护及蝴蝶群落维持。

\section{1 研究区域与研究方法}

\section{1 研究地点选择}

北京市 $\left(115^{\circ} 24^{\prime}-117^{\circ} 30^{\prime} \mathrm{E}, 39^{\circ} 38^{\prime}-41^{\circ} 05^{\prime} \mathrm{N}\right)$ 属 北温带半湿润大陆性季风气候, 四季分明,夏季高温多 雨, 冬季寒冷干燥。大多数蝶类在山地发生, 在城郊平 原地带记录有 30 余种 ${ }^{[19]}, 2017$ 年调查发现北京主城区 公园内有 31 种蝴蝶分布 ${ }^{[20]}$ 。此次研究选择在北京市 六环路内的平原城区开展,沿北-南、西北-东南、西-东、 西南-东北截面, 根据北京市环状分布格局从 2 环到 6 环( UZ1-UZ5), 选择 26 个城市公园 (图 1), 面积从 $1.8-268 \mathrm{hm}^{2}$ 不等。每个环路间区域分布从 2-11 个 公园不等,各公园间至少间隔 $1 \mathrm{~km}$ 。

\section{2 蝴蝶及蜜源植物调查}

为尽可能观测到公园多种生境中蝴蝶的访花行为, 本研究采用样线和样点观测结合的方法。通过植被类 型和管理强度划分, 公园内主要生境类型有草坪、人工 草地、自然更新草地、乔木人工草地、乔木自然更新草 地、乔木裸地、乔灌人工草地、乔灌自然更新草地和灌丛 9 种。根据公园内已有的生境类型, 分别设置 $2-9$ 个

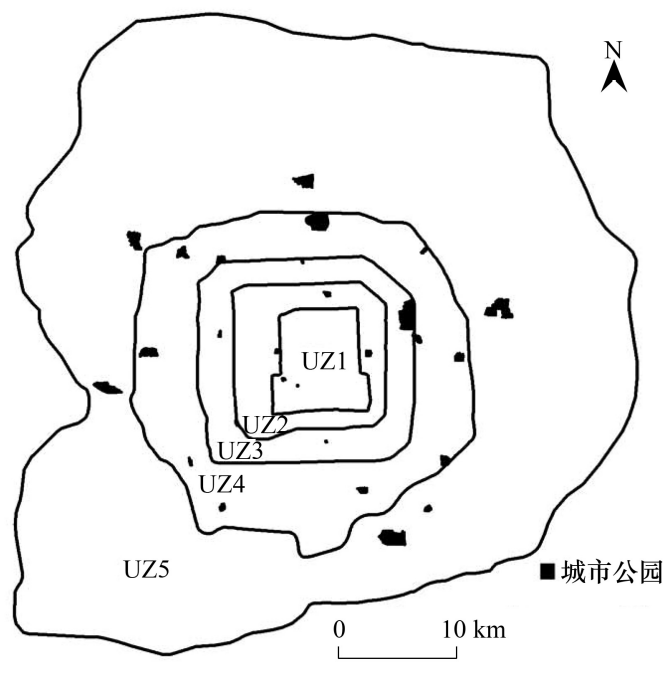

图 1 研究点在北京市六环城区的分布

Fig. 1 Distribution of study sites inside Beijing Six-ring-road urban areas

UZ 为北京环路间不同城市区域 (urban zone): UZ1 为北京 2 环内 城市区域; UZ2 为 2-3 环内城市区域; UZ3 为 3-4 环内城市区 域; UZ4 为 4-5 环内城市区域; UZ5 为 5-6 环内城市区域 $20 \mathrm{~m} \times 20 \mathrm{~m}$ 观测样方。在各公园园内设置 $0.5-9 \mathrm{~km}$ 的样线,使样线观测面积至少占公园绿地面积的 $5 \%$ 以上,样线沿小径分布全园，且各样线间平行间距大于 $40 \mathrm{~m}$ 。在 26 个城市公园中共选取了 $74 \mathrm{~km}$ 样线和 159 个样方。

在访花蝴蝶及蜜源植物调查中, 将嗄伸人花冠中并稍作停留的蝴蝶定义为访花蝴蝶, 所被访问的植物为 蜜源植物, 每只蝴蝶的一次访花视为一次互作。2020 年 6-9 月,选择晴朗温暖无风的天气, 在 8:30-12:00, $15: 00-17: 30$, 沿固定样线以 $1.5 \mathrm{~km} / \mathrm{h}$ 速度匀速前进, 记录行走路线左右 $2.5 \mathrm{~m}$ 、上方 $5 \mathrm{~m}$ 、前方 $5 \mathrm{~m}$ 范围内所 见到的访花蝴蝶种类、蜜源植物种类及互作次数 ${ }^{[21]}$, 在每个 $20 \mathrm{~m} \times 20 \mathrm{~m}$ 的样方内进行 10 分钟观测, 记录访花 蝴蝶种类、蜜源植物种类及互作次数。每间隔 $30 \mathrm{~d}$ 观测一次,遇阴雨天顺延, 累计采样 4 次。现场记录观察到 的蝴蝶种类, 对部分肉眼无法确定种类用昆虫网捕捉鉴定记录后释放,少部分当时无法鉴定种类放人三角纸带 回, 实验室制成标本后, 查阅《中国蝴蝶图鉴》 ${ }^{[22]}$ 、《中国蝶类志》 ${ }^{[23]}$ 、《中国蝴蝶分类与鉴定》 ${ }^{[24]}$ 识别鉴定。

根据植物生长型, 将蜜源植物物种划分为草本型和灌木型, 由于观察高度有限, 本次调查未记录乔木蜜源 植物。根据植物起源, 将蜜源植物物种划分为乡土物种和外来物种。现场判断植物的栽培方式, 将蜜源植物 物种划分为公园内人工栽培物种和自然更新物种。

\section{3 数据分析}

\subsection{1 网络特征指标}

用访花蝴蝶种类、蜜源植物种类以及蝴蝶访问植物的次数, 通过 $\mathrm{R}$ 语言中的 bipartite 包 $^{[25-27]}$, 构建蝴蝶- 
蜜源植物相互作用网络。其中一种植物和一种蝴蝶之间的相互作用视为一种交互,每次访问视为一次交互 (链接), 生成加权网络定量指标。加权网络考虑了不同物种及其伙伴间的交互强度不等,使其定量指标受采 样强度的影响较小。

在网络层级采用 3 个网络特征值定量描述北京蝴蝶-蜜源网络特征 ${ }^{[13]}$ : (1) 交互作用多样性指数 (Interaction diversity, $I D$ ), 即整个网络的加权交互多样性, 为所有链接的 Shannon-Wiener 指数, 较高的值反映 了较高的网络稳定性。(2) 交互作用均匀度指数 (Interaction evenness, $I E$ ), 即网络中所有链接的均匀度指数, 较高的值反映了网络中物种之间相互作用同质性更强, 分布更均匀。(3) 网络专业化指数 (Network

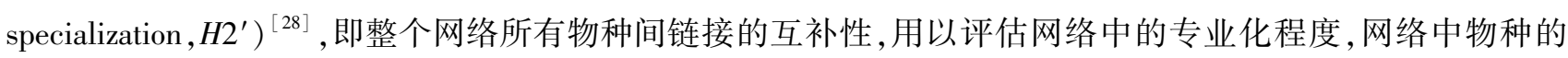
链接选择越少,则网络的专业性越高。 $H 2^{\prime}$ 的范围在 0 到 1.0 之间, 分别代表极端泛化和极端专化。 $H 2^{\prime}$ 在很 大程度上不受网络规模的影响,适用于不同规模网络的比较。具体计算公式如下:

$$
\begin{gathered}
I D=-\sum_{i=1}^{S}\left(p_{i} \ln p_{i}\right) \\
I E=\frac{I D}{\ln S}
\end{gathered}
$$

式中, $S$ 为交互网络中的链接总数, $p_{i}$ 为第 $i$ 链接的个体数占全部个体数的百分比。

$$
\begin{aligned}
& H_{2}=-\sum_{i=1}^{r} \sum_{j=1}^{c}\left(p_{i j} \times \ln p_{i j}\right) \\
& H_{2 \max }=-\sum_{i=1}^{r} \sum_{j=1}^{c}\left(q_{i} q_{j} \times \ln q_{i} q_{j}\right) \\
& H^{\prime}{ }_{2}=\frac{H_{2 \max }-H_{2}}{H_{2 \max }-H_{2 \min }}
\end{aligned}
$$

式中, 双方之间的相互作用通常显示在 $r \times c$ 列联表中, $r$ 行代表一方,如开花植物物种, $c$ 列代表另一方,如传 粉物种。 $p_{i j}$ 为植物物种 $i$ 和传粉物种 $j$ 之间相互作用的次数占总相互作用次数的比例。 $q_{i}$ 为植物物种 $i$ 的所 有互作次数占总相互作用次数的比例, $q_{j}$ 为传粉物种 $j$ 的所有互作次数占总相互作用次数的比例。当给定约 束交互矩阵的行列数时, $H_{2 \max }$ 为 $H_{2}$ 达到其理论最大值, 其中每个 $p_{i j}$ 等于其来自随机相互作用矩阵 $\left(q_{i} \times q_{j}\right)$ 的期 望值, 而其理论最小值 $\left(H_{2 \min }\right)$ 可能接近于零。

在物种层级采用 2 个特征值定量化网络中植物或动物的互相作用特征 ${ }^{[13]}$, 并识别重要蜜源植物物种。 采用伙伴多样性指数识别物种的伙伴丰富程度, 采用物种专业化指数识别物种的专业化程度。(1) 伙伴多样 性指数 (Partner diversity, $P D$ ), 即每个物种的相互作用伙伴的 Shannon-Wiener 指数, 较高的值反映了具有较高 的伙伴多样性。(2) 物种专业化指数 (Species specialization, $\left.d^{\prime}\right)^{[28]}$, 与网络专业化指数 $H 2^{\prime}$ 相对应, 表示某个 物种对应另一层级伙伴的专业化程度, 该指数考虑了物种的伙伴多样性和可用性, 可解释为物种实际交互频 率与零模型的偏差,其中零模型假设所有伙伴间的互作与其可用性成比例。 $d^{\prime}$ 的范围在 0 到 1.0 之间,分别代表 物种的极端泛化和极端专化。例如在传粉网络中, 某一传粉者仅出现在一种常见植物上,该传粉者的专业化程 度较低; 然而当某一传粉者仅出现某几种稀有植物上, 该传粉者的专业化程度很高 ${ }^{[26]}$ 。具体计算公式如下:

$$
P D=-\sum_{i=1}^{S}\left(p_{i} \ln p_{i}\right)
$$

式中, $S$ 是交互网络中的链接数, $p_{i}$ 是第 $i$ 链接的个体数占全部个体数的百分比。

$$
\begin{aligned}
& d_{i}=-\sum_{j=1}^{c}\left(p^{\prime}{ }_{i j} \times \ln \frac{p^{\prime}{ }_{i j}}{q_{j}}\right) \\
& d^{\prime}{ }_{i}=\frac{d_{i}-d_{\min }}{d_{\max }-d_{\min }}
\end{aligned}
$$


式中, $p_{i j}$ 为植物物种 $i$ 和传粉物种 $j$ 之间相互作用的次数占总相互作用次数的比例。 $q_{j}$ 为传粉物种 $j$ 的所有互 作次数占总相互作用次数的比例。当给定约束交互矩阵的行列数时, $d_{\text {max }}$ 为 $d$ 达到其理论最大值, 总次数交互 作用除以植物物种数的自然对数。其中每个 $p_{i j}$ 等于其来自随机相互作用矩阵 $\left(q_{i} \times q_{j}\right)$ 的期望值, 而其理论最 小值 $\left(d_{\text {min }}\right)$ 为零, 即 $p^{\prime}{ }_{i j}=q_{j}$ 时的 $d$ 值。

\subsection{2 数据分析}

采用单因素方差分析 (one-way ANOVA) 比较不同类型蜜源植物对蝴蝶吸引力 (吸引蝴蝶访花的频率) 的 差异。采用 Kruskal-Wallis 秩和检验比较各采样月份内蝴蝶蜜源网络特征值的差异, 以及移除不同类型蜜源 植物后网络特征值的差异。显著性水平均设为 $P<0.05$ 。采用变差分解分析 (Variation partitioning analyses) 区 分不同类型蜜源植物影响蝴蝶-蜜源网络特征值 $\left(I D / I E / H 2^{\prime}\right)$ 的纯效应和共同效应,用韦恩图进行图示。

\section{2 结果与分析}

2.1 蝴蝶-蜜源植物网络结构特征

2.1.1 城市蝴蝶与植物物种构成

2020 年 6-9 月在北京城市公园内共记录到 32 种蝴蝶, 隶属 5 科 26 属,灰蝶科 12 种,蛱蝶科 11 种,粉蝶 科 4 种, 凤蝶科 3 种, 弄蝶科 2 种。同时共记录到 519 种植物, 隶属 96 科 336 属, 其中草本 378 种, 灌木 46 种; 乡土植物 466 种, 外来植物 43 种; 属于自然更新生长有 209 种, 人工栽培生长的有 300 种。

\subsection{2 蜜源网络物种组成}

共记录到 1818 次蝴蝶访花行为, 发现 22 种蝴蝶与 81 种蜜源植物之间的 223 种独特的交互作用 (图 2)。 访花蝴蝶隶属于 5 科 18 属,其中灰蝶科 9 种,蛱蝶科 7 种,粉蝶科 4 种, 凤蝶科 1 种,弄蝶科 1 种。蜜源植物 隶属于 27 科 70 属, 其中属于菊科的蜜源植物最丰富, 含 23 属, 32 种, 其次豆科和唇形科的蜜源植物较丰富, 分别含 10 属, 10 种和 7 属, 9 种。蜜源植物中有 72 种为草本型, 9 种为灌木型; 有 65 种乡土物种, 16 种外来 物种; 27 种属于自然更新生长, 54 种属于人工栽培生长。

\subsection{3 蜜源网络结构特征}

北京城市公园蝴蝶蜜源网络总体交互多样性 $(I D)$ 为 4.14 , 交互均匀性 $(I E)$ 为 0.55 , 网络专业化程度 $\left(H 2^{\prime}\right)$ 为 0.38 , 偏向于泛化网络 (表 1$)$ 。总体上 8 月份北京公园内蝴蝶蜜源网络专业化程度最高, 但仍偏向于 泛化网络。9 月份访花蝴蝶的丰富度最高。蜜源植物丰富度在 6 月份最高, 达 45 种。但各月份间蜜源网络 特征值在统计学上无显著差异, 这表明北京城市蝴蝶蜜源网络整体结构季节性差异小,趋于稳定。

表 1 北京城市公园蝴蝶-蜜源植物网络结构特征值

Table 1 The values of the indices of butterfly-nectar plant network in Beijing' urban parks

\begin{tabular}{|c|c|c|c|c|c|}
\hline 网络特征值 Metric & $\begin{array}{l}\text { 总体 } \\
\text { Total }\end{array}$ & $\begin{array}{l}\text { 6月 } \\
\text { June }\end{array}$ & $\begin{array}{l}7 \text { 月 } \\
\text { July }\end{array}$ & $\begin{array}{c}8 \text { 月 } \\
\text { August }\end{array}$ & $\begin{array}{c}9 \text { 月 } \\
\text { September }\end{array}$ \\
\hline 交互多样性 $I D$ Interaction diversity & 4.14 & 3.60 & 3.54 & 3.63 & 3.18 \\
\hline 交互均匀度 $I E$ Interaction evenness & 0.55 & 0.56 & 0.57 & 0.59 & 0.50 \\
\hline 网络专业化程度 $H 2^{\prime}$ Network specialization & 0.38 & 0.29 & 0.40 & 0.48 & 0.29 \\
\hline 访花蝴蝶丰富度 Butterfly richness & 22 & 13 & 13 & 13 & 16 \\
\hline 蜜源植物丰富度 Nectar plant richness & 81 & 45 & 37 & 37 & 35 \\
\hline
\end{tabular}

2.2 不同类型植物对蝴蝶蜜源网络的影响

2.2.1 不同类型植物对蝴蝶访花的影响

不同生长型、不同起源、不同栽培方式蜜源植物吸引蝴蝶的访花频率均存在极显著差异 $(P<0.05)$ 。72 种 草本植物吸引了 21 种蝴蝶 ( $95.45 \%$ ), 发生了 $95.28 \%$ 的访花。65 种乡土植物支持了全部 22 种蝴蝶的 $71.55 \%$ 的访花行为。54 种人工栽培的植物支持了全部 22 种蝴蝶的 $84.57 \%$ 的访花行为。这表明不同类型的植物对 访花蝴蝶的吸引力不同, 有必要继续探究植物类型对蜜源网络结构的影响。 


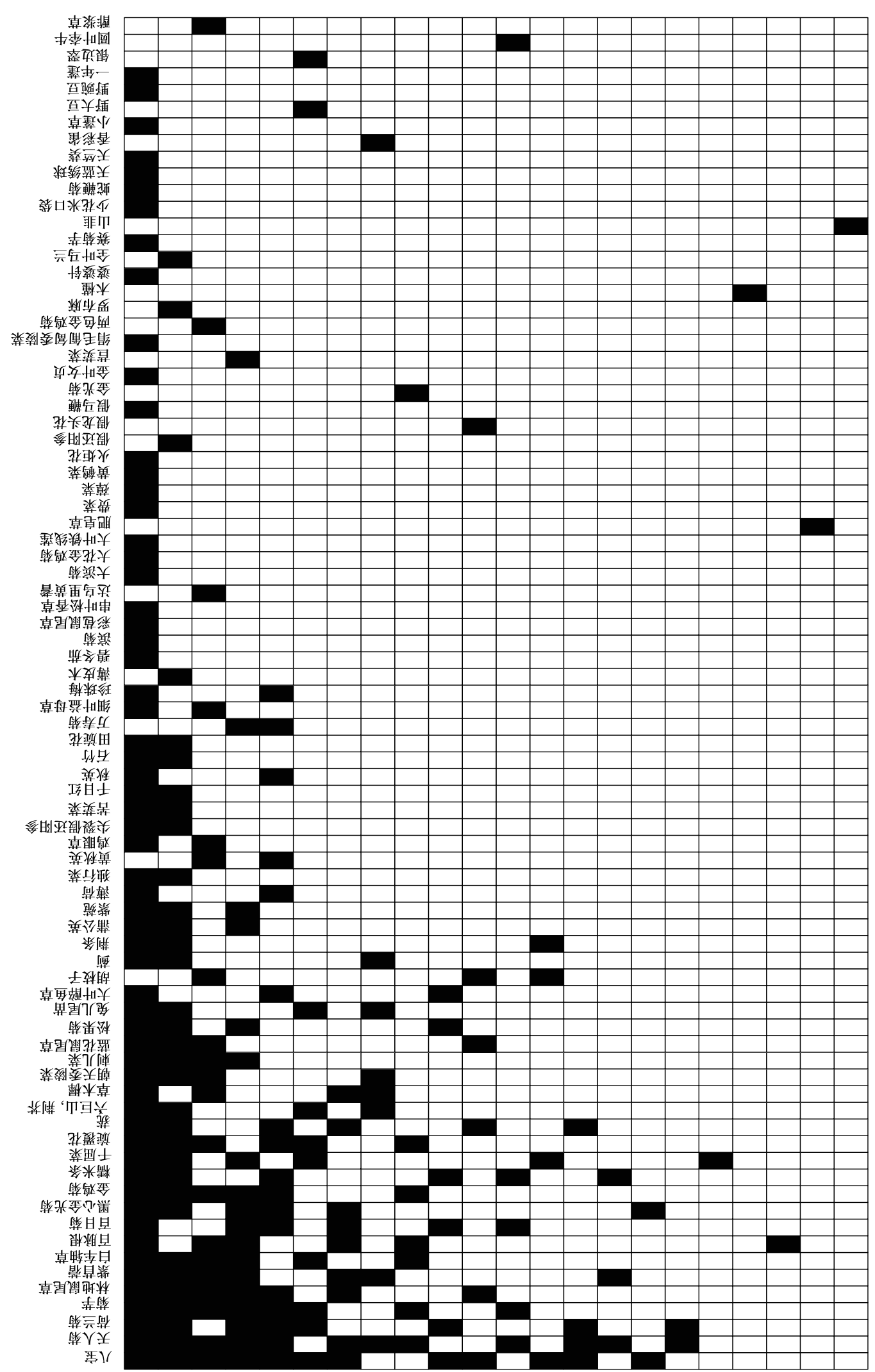

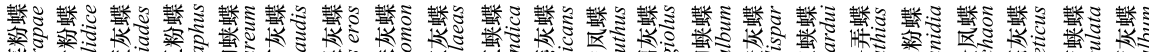

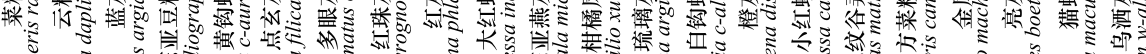

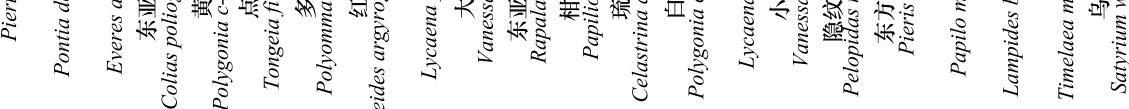




\subsection{2 不同类型植物-蝴蝶蜜源网络}

从总体生态网络中分别移除不同类型蜜源植物后, 改变了二元网络的拓扑结构,仅灌木-蝴蝶网络中专业 化程度 $\left(H 2^{\prime}\right.$ ) 大于 0.5 (表 2), 趋向于专化网络, 而其他类型植物-蝴蝶网络仍表现为泛化网络。进一步从各公 园生态网络中分别移除不同类型植物后,计算网络特征值。结果发现,在交互多样性 $(I D)$ 上,草本-蝴蝶网络 显著高于灌木-蝴蝶网络, 乡土植物-蝴蝶网络显著高于外来植物网络 $(P<0.01)$; 交互均匀性 $(I E)$ 上, 仅有草 本-蝴蝶网络显著高于灌木-蝴蝶网络 $(P<0.01)$; 专业化程度 $\left(H 2^{\prime}\right)$ 上, 草本-蝴蝶网络显著高于灌木-蝴蝶网 络,乡土植物-蝴蝶网络显著高于外来植物网络 $(P<0.01)$ 。而不同栽培方式的植物-蝴蝶生态网络的 3 种网络 指标未存在显著差异, 这表明植物的栽培方式对于蜜源网络结构可能无显著影响。

表 2 不同植物类型-蝴蝶网络的网络层级指标比较

Table 2 Comparison of butterfly and different plant group communities based on indices analysis for networks structured following removal of respective plant group

\begin{tabular}{|c|c|c|c|c|c|c|}
\hline $\begin{array}{l}\text { 网络 } \\
\text { Network }\end{array}$ & & $\begin{array}{c}\text { 交互 } \\
\text { 多样性 } I D \\
\text { Interaction } \\
\text { diversity }\end{array}$ & $\begin{array}{c}\text { 交互 } \\
\text { 均匀性 } I E \\
\text { Interaction } \\
\text { evenness }\end{array}$ & $\begin{array}{c}\text { 网络专业化 } \\
\text { 程度 } H 2^{\prime} \\
\text { Network } \\
\text { specialization }\end{array}$ & $\begin{array}{l}\text { 访花蝴蝶 } \\
\text { 丰富度 } \\
\text { Butterfly } \\
\text { richness }\end{array}$ & $\begin{array}{l}\text { 蜜源植物 } \\
\text { 丰富度 } \\
\text { Nectar plant } \\
\text { richness }\end{array}$ \\
\hline 生长型 & 草本-蝴蝶网络 & 4.02 & 0.55 & 0.37 & 21 & 72 \\
\hline Growth form & 灌木-蝴蝶网络 & 2.70 & 0.58 & 0.54 & 12 & 9 \\
\hline 起源 & 乡土植物-蝴蝶网络 & 4.00 & 0.55 & 0.39 & 22 & 65 \\
\hline Plant origin & 外来植物-蝴蝶网络 & 2.41 & 0.45 & 0.39 & 13 & 16 \\
\hline 栽培方式 & 自然更新植物-蝴蝶网络 & 3.33 & 0.59 & 0.31 & 11 & 27 \\
\hline Plant cultivation & 人工栽培植物-蝴蝶网络 & 3.79 & 0.54 & 0.35 & 22 & 54 \\
\hline
\end{tabular}

\subsection{3 不同类型植物对整体网络的贡献}

通过变差分解分析不同类型蜜源植物对整体生态网络的贡献率。结果表明,北京城市公园蝴蝶蜜源网络 的 3 个网络特征中, 蜜源植物对交互多样性 $(I D)$ 的解释率较高 (图 3 ), 说明蜜源植物的类型对网络交互多样

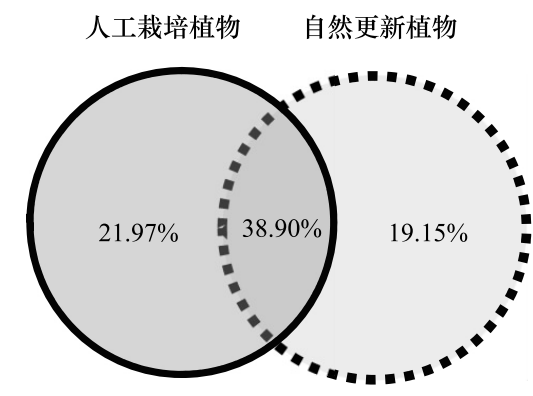

残差 $=25.98 \%$

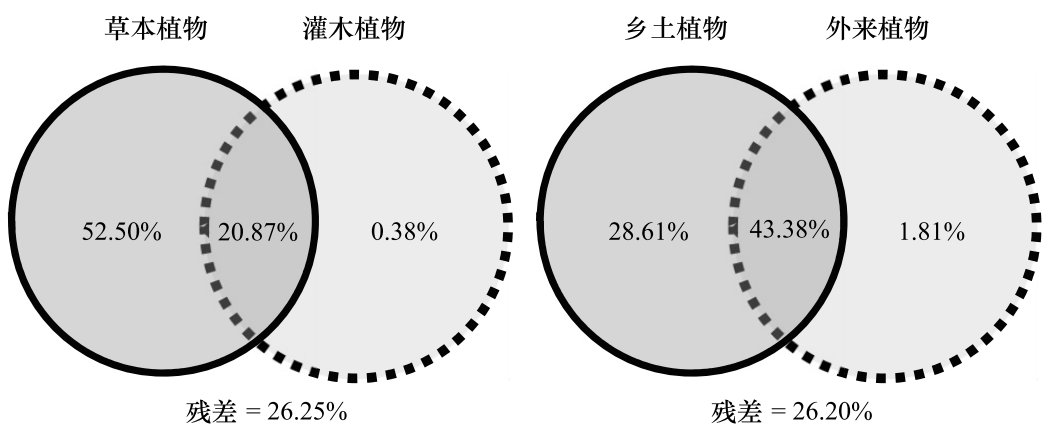

图 3 北京城市公园蝴蝶蜜源网络交互多样性 ID 变差分解

Fig.3 Variance partitioning analysis of nectar plant types affecting interaction diversity of butterfly-nectar plant network in Beijing' urban parks

ID: 交互多样性 Interaction diversity 
性影响较高。而对交互均匀性 (IE) 和网络专业化程度 $\left(H 2^{\prime}\right)$ 的解释率较低 (图 4,5), 其中交互均匀性 $(I E)$ 的 未解释率 (即残差) 高于 $95 \%$ (图 4), 说明蜜源植物类型并不能较好解释网络结构的交互均匀性。

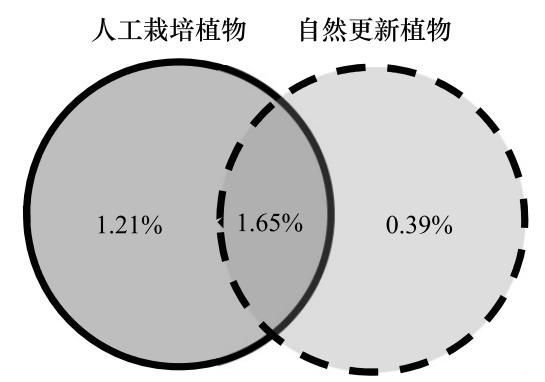

残差 $=96.74 \%$

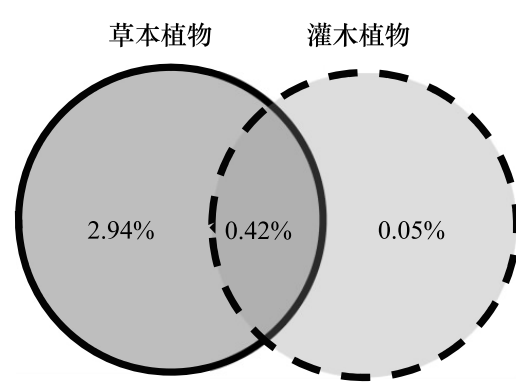

残差 $=96.59 \%$

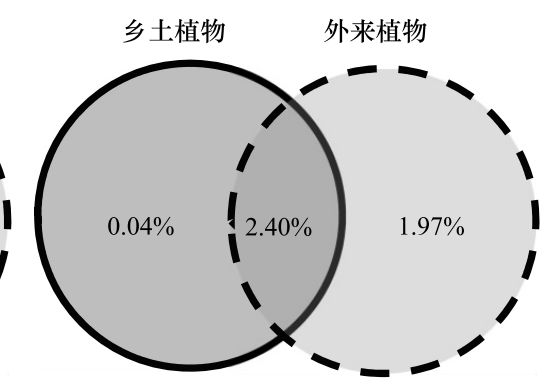

残差 $=95.58 \%$

图 4 北京城市公园蝴蝶蜜源网络交互均匀性 $I E$ 变差分解

Fig.4 Variance partitioning analysis of nectar plant types affecting interaction evenness of butterfly-nectar plant network in Beijing' urban parks

$I E$ : 交互均匀度 Interaction evenness
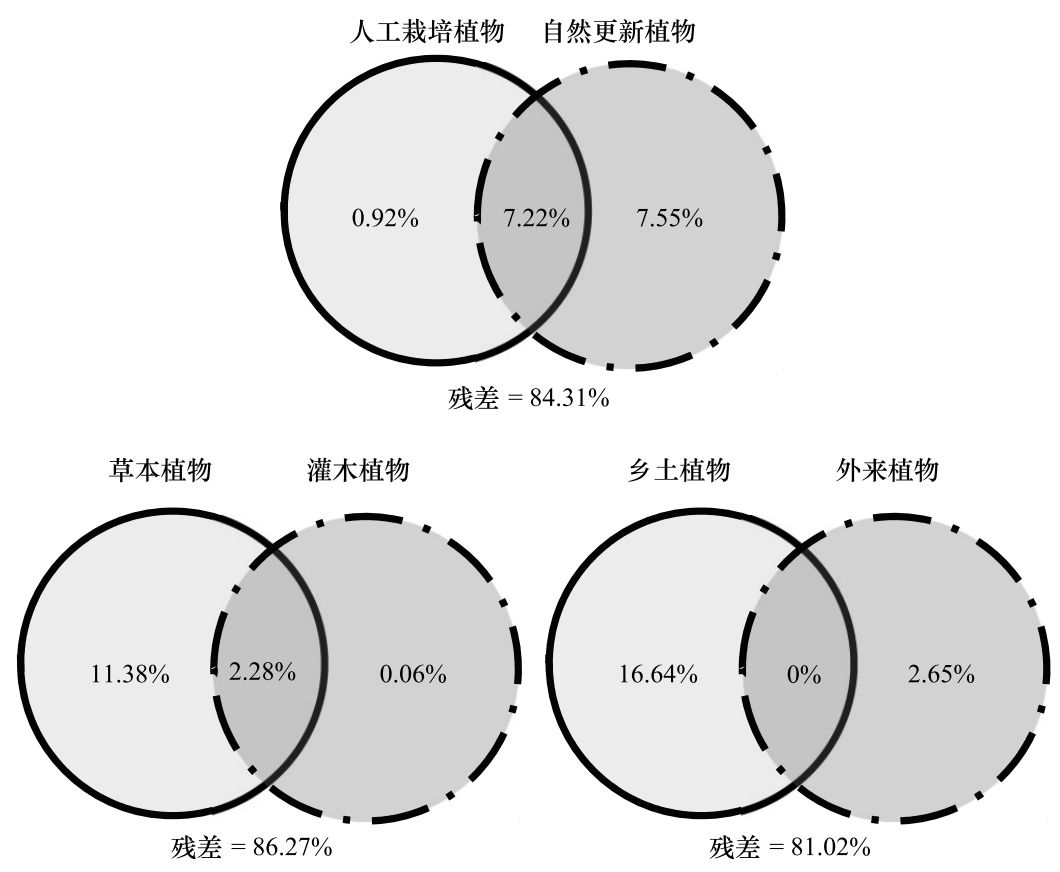

图 5 北京城市公园蝴蝶蜜源网络专业化程度 $H_{2}{ }^{\prime}$ 变差分解

Fig.5 Variance partitioning analysis of nectar plant types affecting network specialization of butterfly-nectar plant network in Beijing' urban parks

$H 2^{\prime}$ : 网络专业化程度 Network specialization 
人工栽培和自然更新两种栽培方式的蜜源植物对网络结构中的交互多样性 (ID) 的共同解释率最高 (38.9\%), 两种栽培方式的蜜源植物各自对交互多样性 (ID) 解释率接近(图 3), 说明人工栽培和自然更新植 物共同支持了网络的交互多样性。草本蜜源植物对网络的交互多样性 (ID) 的解释率最高 $(52.50 \%$ ), 其次是 交叉解释率(20.87\%), 而灌木植物的解释率仅 0.38\% (图 3)。乡土和外来蜜源植物对网络的交互多样性 (ID) 的交叉解释率最高 ( $43.38 \%$ ), 其次是乡土蜜源植物的解释率 ( $28.61 \%$ ), 外来蜜源植物解释率最低仅 $1.81 \%$ (图 3)。这说明草本蜜源对网络交互多样性的贡献率更高, 而单独的灌木以及外来植物不能支持较高 的网络交互性。

自然更新植物对网络专业化程度 $\left(H 2^{\prime}\right)$ 的纯效应 $(7.55 \%)$ 、自然更新与人工栽培植物的共同效应 $(7.22 \%)$ 远高于人工栽培植物的纯效应 $(0.92 \%)$ 。草本植物和乡土植物的纯效应分别对网络专业化程度解 释较高 $(11.38 \%, 16.64 \%$ ) (图 5)。这说明草本植物和乡土植物对维持网络中专业性更高的蝴蝶物种贡献更 大, 而仅有人工栽培植物时并不能较好地支持专化蝴蝶。

2.3 重要蜜源植物物种

菜粉蝶 (Pieris rapae)、云粉蝶 (Pontia daplidice) 、蓝灰蝶 (Everes argiades)、东亚豆粉蝶 (Colias poliographus)、黄钩蛱蝶 (Polygonia c-aureum) 植物伙伴丰富度较高, 即取食较多种蜜源植物, 而东方菜粉蝶 (P. canidia) 等 5 种蝴蝶仅观察到取食 1 种植物 (表 3 )。菜粉蝶、云粉蝶和蓝灰蝶的总体伙伴多样性较高 (表 $3)$ 。根据蝴蝶物种专业化程度 $\left(d^{\prime}\right)$, 金风蝶 (Papilo machaon)、猫蛱蝶 (Timelaea maculata) 和乌酒灰蝶 (Satyrium w-album) 表现出极端专化 $\left(d^{\prime}=1\right)$, 其所进食的植物物种分别为木槿 (Hibiscus syriacus)、肥皇草 (Saponaria officinalis) 和山非(Allium senescens) 也仅被观测到支持这三种蝴蝶(图 2), 从而这三种植物也被视

表 3 北京公园蝴蝶蜜源网络蝴蝶物种特征值

Table 3 Values of the specie level indices for butterfly species of butterfly-nectar plant network in Beijing's urban parks

\begin{tabular}{|c|c|c|c|}
\hline $\begin{array}{l}\text { 蝴蝶 } \\
\text { Butterfly species }\end{array}$ & $\begin{array}{c}\text { 伙伴丰富度 } \\
\text { Partner richness }\end{array}$ & $\begin{array}{l}\text { 伙伴多样性 } P D \\
\text { Partner diversity }\end{array}$ & $\begin{array}{l}\text { 物种专业化指数 } d^{\prime} \\
\text { Species specialization }\end{array}$ \\
\hline 菜粉蝶 Pieris rapae & 61 & 3.12 & 0.30 \\
\hline 云粉蝶 Pontia daplidice & 33 & 3.05 & 0.32 \\
\hline 蓝灰蝶 Everes argiades & 20 & 2.65 & 0.44 \\
\hline 红珠灰蝶 Lycaeides argyrognomon & 8 & 1.95 & 0.37 \\
\hline 红灰蝶 Lycaena phlaeas & 7 & 1.95 & 0.29 \\
\hline 东亚豆粉蝶 Colias poliographus & 18 & 1.92 & 0.23 \\
\hline 黄钩蛱蝶 Polygonia c-aureum & 17 & 1.82 & 0.54 \\
\hline 多眼灰蝶 Polyommatus eros & 9 & 1.76 & 0.39 \\
\hline 大红蛱蝶 Vanessa indica & 6 & 1.52 & 0.25 \\
\hline 东亚燕灰蝶 Rapala micans & 6 & 1.43 & 0.36 \\
\hline 点玄灰蝶 Tongeia filicaudis & 10 & 1.42 & 0.43 \\
\hline 柑橘风蝶 Papilio xuthus & 5 & 1.31 & 0.58 \\
\hline 琉璃灰蝶 Celastrina argiolus & 4 & 1.28 & 0.34 \\
\hline 小红蛱蝶 Vanessa cardui & 4 & 1.17 & 0.17 \\
\hline 白钩蛱蝶 Polygonia c-album & 3 & 0.95 & 0.41 \\
\hline 橙灰蝶 Lycaena dispar & 2 & 0.69 & 0.22 \\
\hline 隐纹谷弄蝶 Pelopidas mathias & 2 & 0.64 & 0.07 \\
\hline 金凤蝶 Papilo machaon & 1 & 0.00 & 1.00 \\
\hline 猫蛱蝶 Timelaea maculata & 1 & 0.00 & 1.00 \\
\hline 乌酒灰蝶 Satyrium w-album & 1 & 0.00 & 1.00 \\
\hline 亮灰蝶 Lampides boeticus & 1 & 0.00 & 0.37 \\
\hline 东方菜粉蝶 Pieris canidia & 1 & 0.00 & 0.12 \\
\hline
\end{tabular}


为极端专化种 $\left(d^{\prime}=1\right)$ (表 4)。东方菜粉蝶的伙伴多样性极低, 但其趋向于泛化种, 专业化程度较低 $\left(d^{\prime}=\right.$ 0.12 ) (表 3), 主要是由于其所访问的千屈菜 (Lythrum salicaria) 较为常见。其它专业化程度较高的物种有柑 橘风蝶 (P.xuthus) 和黄钩蛱蝶, 黄钩蛱蝶虽然访问的植物多达 17 种, 但专业化程度较高 $\left(d^{\prime}=0.54\right)$ (表 3$)$, 这 说明黄钩蛱蝶的部分蜜源植物伙伴较为少见, 并支持较少种蝴蝶。其它专业化程度较低的物种有隐纹谷弄蝶 (Pelopidas mathias) 和小红蛱蝶 (Vanessa cardui) (表 3)，其所访问的蜜源植物如荷兰菊(Aster novi-belgii)、天人 菊 (Gaillardia pulchella) 也较为常见,并支持较多种蝴蝶。

最受蝴蝶访花青睐的植物是天人菊、八宝 (Hylotelephium erythrostictum)、菊芋 (Helianthus tuberosus)、荷兰 菊等,而假马鞭 (Stachytarpheta jamaicensis) 等 40 种的开花植物仅被 1 种蝴蝶取食。糯米条( Abelia chinensis)、 百脉根 (Lotus corniculatus)、八宝、草木灖 (Melilotus officinalis) 的伙伴多样性指数 $(P D)$ 较高, 专业化指数 $\left(d^{\prime}\right)$ 也较高 (表 4), 说明这些植物支持较多种且专业性较高的蝴蝶。反之, 菊芋、黑心金光菊 (Rudbeckia hirta)、百 日菊 (Zinnia elegans) 等植物的伙伴多样性指数 $(P D)$ 较高, 但专业化指数 $\left(d^{\prime}\right)$ 较低 (表 4$)$, 说明这些植物支持 较多种的泛化蝴蝶。少花米口袋 (Gueldenstaedtia verna) 、天答葵 (Pelargonium hortorum) 等 15 种植物的伙伴多 样性指数 $(P D)$ 和专业化指数 $\left(d^{\prime}\right)$ 为 0 (表 4), 是由于其仅被泛化种菜粉蝶访问 (图 2)。而肥皇草、木槿和山 非的专业化指数 $\left(d^{\prime}\right)$ 为 1 (表 4$)$, 分别支持了稀少的极端专化蝴蝶猫蛱蝶、金凤蝶和乌酒灰蝶。

表 4 北京公园蝴蝶蜜源网络蜜源植物物种特征值

Table 4 Values of the specie level indices for nectariferous plant species of butterfly-nectar plant network in Beijing's urban parks

\begin{tabular}{|c|c|c|c|c|}
\hline $\begin{array}{l}\text { 序号 } \\
\text { No. }\end{array}$ & $\begin{array}{l}\text { 蜜源植物 } \\
\text { Plant species }\end{array}$ & $\begin{array}{c}\text { 伙伴丰富度 } \\
\text { Partner richness }\end{array}$ & $\begin{array}{l}\text { 伙伴多样性 } P D \\
\text { Partner diversity }\end{array}$ & $\begin{array}{l}\text { 物种专业化指数 } d^{\prime} \\
\text { Species specialization }\end{array}$ \\
\hline 1 & 糯米条 Abelia chinensis & 6 & 1.48 & 0.43 \\
\hline 2 & 紫苜宿 Medicago sativa & 7 & 1.43 & 0.18 \\
\hline 3 & 白车轴草 Trifolium repens & 6 & 1.41 & 0.16 \\
\hline 4 & 百脉根 Lotus corniculatus & 6 & 1.40 & 0.30 \\
\hline 5 & 天人菊 Gaillardia pulchella & 12 & 1.39 & 0.12 \\
\hline 6 & 八宝 Hylotelephium erythrostictum & 12 & 1.32 & 0.33 \\
\hline 7 & 百日菊 Zinnia elegans & 6 & 1.28 & 0.12 \\
\hline 8 & 金鸡菊 Coreopsis drummondii & 6 & 1.28 & 0.09 \\
\hline 9 & 菊芋 Helianthus tuberosus & 8 & 1.24 & 0.07 \\
\hline 10 & 黑心金光菊 Rudbeckia hirta & 6 & 1.22 & 0.06 \\
\hline 11 & 朝天委陵菜 Potentilla supina & 4 & 1.22 & 0.16 \\
\hline 12 & 松果菊 Echinacea purpurea & 4 & 1.12 & 0.14 \\
\hline 13 & 大叶醉鱼草 Buddleja davidii & 3 & 1.10 & 0.15 \\
\hline 14 & 旋覆花 Inula japonica & 6 & 1.09 & 0.20 \\
\hline 15 & 草木樨 Melilotus officinalis & 4 & 1.08 & 0.34 \\
\hline 16 & 蒲公英 Taraxacum mongolicum & 3 & 1.05 & 0.11 \\
\hline 17 & 获 Caryopteris divaricata & 6 & 1.04 & 0.12 \\
\hline 18 & 荷兰菊 Aster novi-belgii & 8 & 1.03 & 0.22 \\
\hline 19 & 刺儿菜 Cirsium setosum & 4 & 1.01 & 0.09 \\
\hline 20 & 蓟 Cirsium japonicum & 3 & 0.86 & 0.17 \\
\hline 21 & 林地鼠尾草 Salvia nemorosa & 7 & 0.78 & 0.17 \\
\hline 22 & 荆条 Vitex negundo & 3 & 0.74 & 0.13 \\
\hline 23 & 胡枝子 Lespedeza bicolor & 3 & 0.72 & 0.59 \\
\hline 24 & 紫苑 Aster tataricus & 3 & 0.72 & 0.15 \\
\hline 25 & '六巨山' 荆芥 Nepeta $\times$ faassenii 'Six Hills Giant' & 4 & 0.71 & 0.14 \\
\hline 26 & 千日红 Gomphrena globosa & 2 & 0.69 & 0.09 \\
\hline 27 & 鸡眼草 Kummerowia striata & 2 & 0.69 & 0.15 \\
\hline 28 & 细叶益母草 Leonurus sibiricus & 2 & 0.69 & 0.15 \\
\hline
\end{tabular}




\begin{tabular}{|c|c|c|c|c|}
\hline 序号 & 蜜源植物 & 伙伴丰富度 & 伙伴多样性 $P D$ & 物种专业化指数 $d^{\prime}$ \\
\hline & & & & Species specialization \\
\hline 29 & 尖裂假还阳参 Crepidiastrum sonchifolium & 2 & 0.69 & 0.25 \\
\hline 30 & 秋英 Cosmos bipinnata & 2 & 0.67 & 0.02 \\
\hline 31 & 苦实菜 Ixeris polycephala & 2 & 0.67 & 0.15 \\
\hline 32 & 蓝花鼠尾草 Salvia farinacea & 4 & 0.66 & 0.14 \\
\hline 33 & 石竹 Dianthus chinensis & 2 & 0.66 & 0.11 \\
\hline 34 & 兔儿尾苗 Veronica longifolia & 4 & 0.66 & 0.12 \\
\hline 35 & 珍珠梅 Sorbaria sorbifolia & 2 & 0.50 & 0.07 \\
\hline 36 & 田旋花 Convolvulus arvensis & 2 & 0.50 & 0.11 \\
\hline 37 & 万寿菊 Tagetes erecta & 2 & 0.45 & 0.17 \\
\hline 38 & 黄秋英 Cosmos sulphureus & 2 & 0.38 & 0.25 \\
\hline 39 & 独行菜 Lepidium apetalum & 2 & 0.35 & 0.35 \\
\hline 40 & 薄荷 Mentha canadensis & 2 & 0.32 & 0.21 \\
\hline 41 & 千屈菜 Lythrum salicaria & 6 & 0.22 & 0.26 \\
\hline 42 & 碧冬茄 Petunia hybrida & 1 & 0.00 & 0.00 \\
\hline 43 & 滨菊 Leucanthemum vulgare & 1 & 0.00 & 0.00 \\
\hline 44 & 串叶松香草 Silphium perfoliatum & 1 & 0.00 & 0.00 \\
\hline 45 & 大滨菊 Leucanthemum maximum & 1 & 0.00 & 0.00 \\
\hline 46 & 大叶铁线莲 Clematis heracleifolia & 1 & 0.00 & 0.00 \\
\hline 47 & 蔊菜 Rorippa indica & 1 & 0.00 & 0.00 \\
\hline 48 & 火炬花 Kniphofia uvaria & 1 & 0.00 & 0.00 \\
\hline 49 & 绢毛匍甸委陵菜 Potentilla reptans var. sericophylla & 1 & 0.00 & 0.00 \\
\hline 50 & 赛菊芋 Heliopsis helianthoides & 1 & 0.00 & 0.00 \\
\hline 51 & 少花米口袋 Gueldenstaedtia verna & 1 & 0.00 & 0.00 \\
\hline 52 & 蛇鞭菊 Liatris spicata & 1 & 0.00 & 0.00 \\
\hline 53 & 天蓝绣球 Phlox paniculata & 1 & 0.00 & 0.00 \\
\hline 54 & 天竺葵 Pelargonium hortorum & 1 & 0.00 & 0.00 \\
\hline 55 & 小蓬草 Conyza canadensis & 1 & 0.00 & 0.00 \\
\hline 56 & 野踠豆 Vicia sepium & 1 & 0.00 & 0.00 \\
\hline 57 & 金叶女贞 Ligustrum×vicaryi & 1 & 0.00 & 0.07 \\
\hline 58 & 婆婆针 Bidens bipinnata & 1 & 0.00 & 0.07 \\
\hline 59 & 一年蓬 Erigeron annuиs & 1 & 0.00 & 0.07 \\
\hline 60 & 大花金鸡菊 Coreopsis grandiflora & 1 & 0.00 & 0.08 \\
\hline 61 & 彩苍鼠尾草 Salvia viridis & 1 & 0.00 & 0.09 \\
\hline 62 & 费菜 Sedum aizoon & 1 & 0.00 & 0.09 \\
\hline 63 & 黄鹤菜 Youngia japonica & 1 & 0.00 & 0.10 \\
\hline 64 & 假马鞭 Stachytarpheta jamaicensis & 1 & 0.00 & 0.12 \\
\hline 65 & 罗布麻 Apocynum venetum & 1 & 0.00 & 0.25 \\
\hline 66 & 全叶马兰 Aster pekinensis & 1 & 0.00 & 0.25 \\
\hline 67 & 薄皮木 Leptodermis oblonga & 1 & 0.00 & 0.33 \\
\hline 68 & 假还阳参 Crepidiastrum lanceolatum & 1 & 0.00 & 0.33 \\
\hline 69 & 苣实菜 Sonchus arvensis & 1 & 0.00 & 0.35 \\
\hline 70 & 两色金鸡菊 Coreopsis tinctoria & 1 & 0.00 & 0.37 \\
\hline 71 & 酢浆草 Oxalis corniculata & 1 & 0.00 & 0.37 \\
\hline 72 & 达乌里黄耆 Astragalus dahuricus & 1 & 0.00 & 0.45 \\
\hline 73 & 野大豆 Glycine soja & 1 & 0.00 & 0.49 \\
\hline 74 & 银边翠 Euphorbia marginata & 1 & 0.00 & 0.49 \\
\hline
\end{tabular}




\begin{tabular}{clccc} 
续表 & \multicolumn{3}{c}{} \\
\hline 序号 & 蜜源植物 & $\begin{array}{c}\text { 伙伴丰富度 } \\
\text { Partner richness }\end{array}$ & $\begin{array}{c}\text { 伙伴多样性 } P D \\
\text { Partner diversity }\end{array}$ & $\begin{array}{c}\text { 物种专业化指数 } d^{\prime} \\
\text { Species specialization }\end{array}$ \\
\hline No. & Plant species & 1 & 0.00 & 0.61 \\
75 & 香彩雀 Angelonia angustifolia & 1 & 0.00 & 0.63 \\
76 & 圆叶冢牛 Pharbitis purpurea & 1 & 0.00 & 0.67 \\
77 & 假龙头花 Physostegia virginiana & 1 & 0.00 & 0.71 \\
78 & 金光菊 Rudbeckia laciniata & 1 & 0.00 & 1.00 \\
79 & 肥㿝草 Saponaria officinalis & 1 & 0.00 & 1.00 \\
80 & 木槿 Hibiscus syriacus & 1 & 0.00 & 1.00 \\
\hline 81 & 山非 Allium senescens & & & \\
\hline
\end{tabular}

\section{3 讨论}

本研究基于城市园林里现有的植物类型和植物种类中去识别蜜源植物及蝴蝶访花行为,在北京城市公园 中共观察到 22 种蝴蝶与 81 种开花植物的交互作用 (图 2), 首次在国内城市生态系统中构建了定量的蝴蝶蜜源植物生态网络。植物丰富度和蜜源植物丰度已被证实对北京城市公园的蝴蝶多样性起决定作用 ${ }^{[29]}$, 而 针对具体的城市蝴蝶群落保护目标, 蝴蝶-植物生态网络能进一步帮助理解不同植物类型和植物物种的作用。

我们的研究结果表明不同类型植物对蝴蝶蜜源网络结构特征的影响有显著差异。城市化过程中自然残 留斑块的去除在一定程度上降低了草本植物的丰富度和丰度 ${ }^{[30]}$, 对城市中草地蝴蝶的多样性产生消极影 响 ${ }^{[31]}$ 。正如我们的研究结果, 相比灌木, 北京城市公园中的草本-蝴蝶网络支持更丰富的物种交互作用 (图 3), 网络专业性更高 (图 5), 能支持更多专业化程度较高的蝴蝶。因此, 城市森林建设过程中需要关注草 本植物群落, 有利于营建蝴蝶的潜在生境。受观测高度限制, 我们未能记录乔木蜜源植物。然而, 我们在实际 调查中也观察到柳紫闪蛱蝶 (Apatura ilia) 吸食白蜡 (Fraxinus chinensis) 树液, 虽然并未纳人本次的生态网络 分析中, 但乔木树种除作为食源外, 也作为多种蝴蝶的寄主植物, 不能忽视其重要性。不同栽培方式的植物蝴蝶网络特征差异不显著,而且人工栽培和自然更新植物的共同作用对蝴蝶蜜源网络交互的多样性影响最大 (图 3), 这说明,城市公园中的人工种植和栽培植物并非总对蝴蝶多样性产生负面影响。但自然更新的植物 支持更高的蝴蝶网络专业化程度 (图 5), 保留一定的原生植物有助于吸引专化蝴蝶。部分研究发现外来植物 物种的人侵会导致本地蝴蝶多样性下降 ${ }^{[32-33]}$ 。由于城市中生境破碎化, 传粉者群落可能会同质性更强, 外来 引进物种有可能替代本土物种, 并主导生态网络中的相互作用 ${ }^{[5]}$ 。本研究发现, 单独的乡土植物-蝴蝶网络比 外来植物-蝴蝶网络更加稳定, 而乡土与外来植物的共同作用对蝴蝶蜜源网络的交互多样性影响最大(图 3), 乡土植物能支持更高的蝴蝶蜜源网络专业化程度 (图 5)。由此可推断, 乡土植物及自然更新植物在本地区长 期存在, 其所构建的植物群落稳定性较强, 能与蝴蝶形成长期稳定的联系。城市森林建设过程中应广泛使用 乡土植物, 保留一定的自然更新植物, 以期营建更加稳定的蝴蝶蜜源网络。但部分人工栽培的外来植物由于 花蜜资源丰富, 能吸引大量泛化蝴蝶, 可作为蝴蝶的补充食源, 吸引蝴蝶成虫聚集,构建临时生境。

我们通过物种的伙伴多样性和专业化程度来识别重要蜜源植物的方法可以用来解决具体的管理目标, 例 如为目标群体最大限度地提供植物资源, 提高其栖息地质量保护。在城市公园中提高植物多样性有利于保护 蝴蝶多样性 ${ }^{[29]}$, 但在条件受限的情况, 若分别针对以下目标, 可以有不同的蜜源植物选择策略: (1) 为构建相 互作用丰富, 且存在特化蝴蝶的稳定蜜源网络结构, 优先选择伙伴多样性指数高, 且专业化指数高的蜜源植 物; (2) 为进一步吸引和支持专业化程度高的蝴蝶, 可增加种植伙伴多样性指数低, 专业化指数高的蜜源植 物; (3) 为吸引数量较多的成虫访问,形成“留连戏蝶时时舞”、“花丛乱数蝶” 的景观效果, 可集中种植伙伴多 样性高, 但专业性低的蜜源植物。蝴蝶成虫集聚飞行的场景在一定程度上内能提高城市公园的生物观赏性, 激发人们对保护城市昆虫等生物的公众意识。我们的研究为城市绿地中蜜源植物的选择提供了科学策略, 也 为北京城市地区提供了具体的蜜源植物物种清单, 显示了构建蜜源生态网络作为保护城市生态系统中生物多 
样性的有效工具的潜力。

虽然北京城市公园整体网络结构季节变化不显著,较为稳定,并趋向于泛化网络 (表 1), 但不同月份网络 中蝴蝶、植物的物种组成有所不同。因此,进一步进行季节性特定蜜源植物重要性的识别, 更能为蝴蝶物种保 育提供季节针对性的实践保护策略。本研究受调查时间和人力限制, 观测时间间隔较长, 未能分别构建相对 稳定的季节性蝴蝶-植物网络。在未来研究中, 应根据植物物候缩短观测间隔时间, 增加观测频率, 并利用多 年积累数据构建季节性生态网络。此外, 部分稀少蝴蝶物种及其访花行为较少被观察到, 在加权网络中存在 一定的偏差, 从而被认为起次要作用, 例如, 金风蝶等蝴蝶仅被观察到一次访花行为, 则易被判定成高度专业 化的物种。在未来生态网络监测中, 在成本和时间投人允许的情况下, 应加大对特殊和稀有城市蝴蝶物种的 监测, 从而可充分论证稀少物种在传粉网络中实际上形成的访问链接。

\section{4 结论}

我们构建了北京城市公园中的实际蝴蝶-蜜源植物生态网络, 分析了蜜源植物类型对网络结构的影响以 及物种在该网络中的地位。结果表明城市公园中乡土草本植物支持更高的蝴蝶多样性和交互作用多样性, 更 能支持专业化程度高的蝴蝶物种, 形成交互作用丰富且趋向专化的生态网络。而人工栽培和自然更新的植物 对蜜源网络结构影响较小。由此, 具有丰富花蜜的人工栽培的外来植物可作为重要的补充性蜜源, 但乡土草 本植物更有可能维持稳定的蝴蝶种群。蝴蝶与蜜源植物的交互特点分析表明伙伴多样性高且专业化程度高 的植物可被视为重要蜜源植物。本研究关于蝴蝶-蜜源植物生态网络的研究, 能为具体物种保护和种群维持 中植物选择提供科学策略,也印证该方法可以成为联结生态研究和城市绿地实践管理的有效工具。未来研究 中蝴蝶植物生态网络需在蜜源网络基础上, 涵盖更多蝴蝶生活阶段, 构建纳人寄主植物的生态网络, 能进一步 理解物种共存, 构建更加稳定的群落结构, 提高城市生态系统服务功能。

致谢:感谢布冰参与蝴蝶观测、段文军博士和孙振凯博士帮助写作。

\section{参考文献 (References) :}

[1] 方强, 黄双全. 传粉网络的研究进展: 网络的结构和动态. 生物多样性, 2012, 20(3) : 300-307.

[ 2 ] Montoya J M, Pimm S L, Solé R V. Ecological networks and their fragility. Nature, 2006, 442(7100): 259-264.

[ 3 ] Bascompte J. Disentangling the web of life. Science, 2009, 325(5939) : 416-419.

[ 4 ] Santamaría L, Rodríguez-Gironés M A. Linkage rules for plant-pollinator networks: trait complementarity or exploitation barriers? PLoS Biology, 2007, 5(2): $\mathrm{e} 31$.

[ 5 ] Hagen M, Kissling W D, Rasmussen C, De Aguiar M A M, Brown L E, Carstensen D W, Alves-Dos-Santos I, Dupont Y L, Edwards F K, Genini J, Guimarães Jr P R, Jenkins G B, Jordano P, Kaiser-Bunbury C N, Ledger M E, Maia K P, Marquitti F M D, Mclaughlin Ó, Morellato L P C, O'Gorman E J, Trøjelsgaard K, Tylianakis J M, Vidal M M, Woodward G, Olesen J M. Biodiversity, species interactions and ecological networks in a fragmented world. Advances in Ecological Research, 2012, 46: 89-210.

[ 6 ] Moragues E, Traveset A. Effect of Carpobrotus spp. on the pollination success of native plant species of the Balearic Islands. Biological Conservation, 2005, 122(4): 611-619.

[ 7 ] Ferrer-Paris J R, Sánchez-Mercado A, Viloria Á L, Donaldson J. Congruence and diversity of butterfly-host plant associations at higher taxonomic levels. PLoS One, 2013, 8(5): e63570.

[ 8 ] Curtis R J, Brereton T M, Dennis R L H, Carbone C, Isaac N J B. Butterfly abundance is determined by food availability and is mediated by species traits. Journal of Applied Ecology, 2015, 52(6) : 1676-1684.

[ 9 ] Dennis R L H, Shreeve T G, Van Dyck H. Habitats and resources: the need for a resource-based definition to conserve butterflies. Biodiversity \& Conservation, 2006, 15(6): 1943-1966.

[10] Nielsen A B, van den Bosch M, Maruthaveeran S, van den Bosch C K. Species richness in urban parks and its drivers: a review of empirical evidence. Urban Ecosystems, 2014, 17(1) : 305-327.

[11] Mukherjee S, Banerjee S, Basu P, Saha G K, Aditya G. Butterfly-plant network in urban landscape: implication for conservation and urban greening. Acta Oecologica, 2018, $92: 16-25$.

[12] Fründ J, Linsenmair K E, Blüthgen N. Pollinator diversity and specialization in relation to flower diversity. Oikos, 2010, 119(10): 1581-1590.

[13] Kaiser-Bunbury C N, Blüthgen N. Integrating network ecology with applied conservation: a synthesis and guide to implementation. AoB PLANTS, 2015, 7: plv076.

[14] Memmott J. Food webs: a ladder for picking strawberries or a practical tool for practical problems? Philosophical Transactions of the Royal Society 
B: Biological Sciences, 2009, 364(1524): 1693-1699.

[15] Russo L, DeBarros N, Yang S, Shea K, Mortensen D. Supporting crop pollinators with floral resources: network-based phenological matching. Ecology and Evolution, 2013, 3(9): 3125-3140.

[16] Aidar I F, Bartelli B F, Nogueira-Ferreira F H. Network of bee-plant interactions and recognition of key species in semideciduous forest. Sociobiology, 2015, 62(4) : 583-592.

[17］杜巍, 王红侠, 汪小凡. 神农架地区典型草本群落中的昆虫访花行为比较. 生物多样性, 2007, 15(6) : 666-672.

[18] Gong Y B, Huang S Q. Temporal stability of pollinator preference in an alpine plant community and its implications for the evolution of floral traits. Oecologia, 2011, 166(3): 671-680.

[19] 杨宏, 王春浩, 禹平. 北京蝶类原色图鉴. 北京: 科学技术文献出版社, 1994.

[20] Sing K W, Luo J S, Wang W Z, Jaturas N, Soga M, Yang X Z, Dong H, Wilson J J. Ring roads and urban biodiversity: distribution of butterflies in urban parks in Beijing city and correlations with other indicator species. Scientific Reports, 2019, 9(1) : 7653.

[21] Pollard E, Yates T J. Monitoring Butterflies for Ecology and Conservation: the British Butterfly Monitoring Scheme. London: Chapman \& Hall, 1993.

[22］武春生, 徐堉峰. 中国蝴蝶图鉴. 福州: 海峡书局, 2017.

[23] 周尧. 中国蝶类志. 郑州: 河南科学技术出版社, 1994.

[24] 周尧. 中国蝴蝶分类与鉴定. 郑州: 河南科学技术出版社, 1998.

[25] Dormann C F, Gruber B, Fründ J. Introducing the bipartite package: analysing ecological networks. R News, 2008, 8(2): 8-11.

[26] Dormann C F, Fründ J, Blüthgen N, Gruber B. Indices, graphs and null models: analyzing bipartite ecological networks. The Open Ecology Journal, 2009, 2: 7-24.

[27] Dormann C. F. How to be a specialist? Quantifying specialisation in pollination networks. Network Biology, 2011, 1(1): 1-20.

[28] Blüthgen N, Menzel F, Blüthgen N. Measuring specialization in species interaction networks. BMC Ecology, 2006, 6: 9.

[29] Han D, Zhang C, Wang C, She J Y, Sun Z K, Zhao D X, Bian Q, Han W J, Yin L Q, Sun R L, Wang X Y, Cheng H. Differences in response of butterfly diversity and species composition in urban parks to land cover and local habitat variables. Forests, 2021, 12(2): 140.

[30] Cameron G N, Culley T M, Kolbe S E, Miller A I, Matter S F. Effects of urbanization on herbaceous forest vegetation: the relative impacts of soil, geography, forest composition, human access, and an invasive shrub. Urban Ecosystem, 2015, 18(4): 1051- 1069.

[31] Konvicka M, Kadlec T. How to increase the value of urban areas for butterfly conservation? A lesson from Prague nature reserves and parks. European Journal of Entomology, 2011, 108(2) : 219-229.

[32] Skórka P, Settele J, Woyciechowski M. Effects of management cessation on grassland butterflies in southern Poland. Agriculture, Ecosystems \& Environment, 2007, 121(4): 319-324.

[33] Florens F B V, Mauremootoo J R, Fowler S V, Winder L, Baider C. Recovery of indigenous butterfly community following control of invasive alien plants in a tropical island's wet forests. Biodiversity and Conservation, 2010, 19(14) : 3835-3848. 